\title{
Surgical site infection prevention
}

Keywords: surgical site infection, hospital acquired infection, LMIC, ABHR, PNPWT

\section{Introduction}

Surgical site infection (SSI) is the most frequent type of hospital acquired infection (HAI) in low- and middle-income countries (LMIC) and affects up to one third of patients who have undergone a surgical procedure. In LMICs, the pooled incidence of SSI was 11.8per 100 surgical procedures (range 1.2 to 23.6). Although SSI incidence is much lower in high-income countries, it remains the second most frequent type of HAI and poses a significant burden in terms of patient morbidity and mortality and additional costs to health systems worldwide.

Numerous risk factors have been identified for the development of an SSI after surgery which are broadly divided into intrinsic (patient) and extrinsic factors (Table 1).

Table I Surgical Site Infection Risk Factors
Volume 4 Issue I - 2018

\section{Syed Nawaz Ahmad,' Richa Aggarwal'}

'Senior Resident, ESIC-PGIMSR, India

${ }^{2}$ Assistant Professor, Department of Obstetrics and Gynecology, University College of Medical Sciences and Guru Teg Bahadur Hospital, Delhi

Correspondence: Richa Aggarwal, Assistant Professor, Department of Obstetrics and Gynecology, University College of Medical Sciences and Guru Teg Bahadur Hospital, Delhi, India, Email richa_agg2005@rediffmail.com

Received: November 10, 2017| Published: January 08, 2018

\section{Risk Factor}

\begin{tabular}{l} 
Non-modifiable \\
Increased age \\
Recent radiotherapy \\
History of skin or soft tissue infection \\
Modifiable \\
Diabetes \\
Obesity \\
related) \\
Alcoholism \\
Current smoker \\
Prictient- $\quad$ Preoperative albumin $<3.5 \mathrm{mg} / \mathrm{dL}$ \\
\hline
\end{tabular}


Table Continued...

\begin{tabular}{ll} 
Risk Factor & \\
\hline Procedure \\
\hline Emergency \\
Increasing complexity \\
Higher wound classification \\
Facility \\
Inadequate ventilation \\
Increased operating room traffic \\
Contaminated environmental surfaces \\
Non-sterile equipment \\
Preoperative \\
Pre-existing infection \\
Inadequate skin preparation \\
Inappropriate antibiotic choice, timing, and weight- \\
based dosing \\
Hair removal method \\
Poor glycemic control \\
Intraoperative \\
Longer procedure duration \\
Blood transfusion \\
Breach in asepsis \\
Inappropriate antibiotic re-dosing \\
Inadequate gloving \\
Inappropriate surgical scrub \\
\hline
\end{tabular}

\section{The CDC provides definitions for the 4 wou- nd classes}

Class I/Clean refers to an uninfected operative wound in which no inflammation is encountered and the alimentary, genital or uninfected urinary tracts are not entered. In addition, clean wounds are primarily closed and, if necessary, drained with closed drainage. Class II/Cleancontaminated refers to operative wounds in which the alimentary, genital or urinary tracts are entered under controlled conditions and without unusual contamination. Specifically, operations involving the appendix and vagina are included in this category, provided no evidence of infection or major break in technique is encountered.

Class III/Contaminated refers to operations with major breaks in sterile technique or gross spillage from the gastrointestinal tract, and incisions in which acute, non-purulent inflammation is encountered. Class IV/Dirty or infected includes operative sites involving existing clinical infection or perforated viscera. This definition suggests that the organisms causing postoperative infection were present in the operative field before the operation.
Various guidelines and evidence-based recommendations have been provided by various organizations including NICE, CDC, WHO etc. to prevent surgical site infection. A summary of recommendations is listed below.

\section{Preoperative measures \\ Preoperative bathing}

The patients should be advised to shower or have a bath using soap, either the day before, or on the day of, surgery. Moderate quality evidence shows that preoperative bathing with antimicrobial soap containing chlorhexidine gluconate has neither benefit nor harm compared to plain soap in reducing the SSI rate, hence either plain or antimicrobial soap may be used.

\section{Nasal decontamination}

S. Aureus is the leading health care-associated pathogen in hospitals worldwide. However, large proportion of HAI due to S. Aureus originate from the patients' own flora. Nasal carriage of 
S. Aureus is considered a well-defined risk factor for subsequent infection in various patient groups. WHO suggests considering to treat patients with known nasal carriage of $S$. Aaureus undergoing surgery with Perioperative intranasal applications of mupirocin $2 \%$ ointment. Nasal decontamination with topical antimicrobials should not be used routinely to reduce the risk of surgical site infection.

\section{Smoking cessation}

Smoking cessation 4 to 6 weeks before surgery reduces SSI and is recommended for all current smokers, especially those undergoing procedures with implanted materials.

\section{Mechanical bowel preparation}

Do not use mechanical bowel preparation routinely to reduce the risk of surgical site infection. Occasionally, there may be risk of bowel injury because of pelvic adhesions resulting from either previous surgery or an inflammatory process, such as PID or endometriosis. In such cases, it is reasonable to consider using a prophylactic parenteral antibiotic regime preoperatively that is effective in preventing infections in patients undergoing elective bowel surgery. There is no evidence that mechanical bowel preparation or addition of oral antibiotics further reduces infection risk.

\section{Antibiotic prophylaxis}

The literature generally supports the administration of prophylactic antibiotics within lhour before incision, or within 2 hours for vancomycin or fluoroquinolones. The antibiotic dosing should be adjusted based on the patient's weight. Antibiotics should be redosed (if appropriate) for any surgical procedure lasting longer than 2-3hours or when substantial blood loss (greater than $1,500 \mathrm{~mL}$ ) occurs. A general rule is that antibiotics should be redosed at one to two times the half-life of the drug measured from the time the preoperative dose is administered. Administer the appropriate parenteral prophylactic antimicrobial agents before skin incision in all cesarean section procedures. Use the local antibiotic formulary and always consider potential adverse effects when choosing specific antibiotics for prophylaxis.

\section{Hair removal}

According to $\mathrm{CDC}$, hair in the surgical site should only be removed if it will interfere with surgery. Shaving causes microscopic cuts and abrasions, resulting in disruption of the skin's barrier defense against microorganisms. As such, razors are no longer recommended. If hair has to be removed, electric clippers with a single-use head should be used on the day of surgery.

\section{Surgical hand scrub}

The WHO guidelines on hand hygiene in health care recommends keeping nails short and to remove all jewellery, artificial nails or nail polish before surgical hand preparation. Surgical hand antisepsis should be performed using either (but not combined) a suitable antimicrobial soap or Alchol based hand rub (ABHR), preferably with a product ensuring sustained activity.

The operating team should wash their hands prior to the first operation on the list using an aqueous antiseptic surgical solution, with a single-use brush or pick for the nails, and ensure that hands and nails are visibly clean. Before subsequent operations, hands should be washed using either an alcoholic hand rub or an antiseptic surgical solution. If hands are soiled, they should be washed again with an antiseptic surgical solution because although alcohol rapidly kills microorganisms, it does not physically remove organic material. Studies have shown that waterless chlorhexidine scrub is as effective as traditional water-based scrubs and requires less time and having a persistent suppressive effect against bacterial regrowth on the skin, potentially lasting throughout several operations. ${ }^{1,2}$

The guidelines stipulate that if the quality of water is not assured in the OR, surgical hand antisepsis using ABHR is recommended. A sufficient amount of ABHR should be applied to dry hands and forearms for the length of time recommended by the manufacturer, typically 1.5 minutes, and hands and forearms allowed to dry before donning sterile gloves.

\section{Surgical attire}

The guidelines call for wearing clean, facility-laundered scrubs that should be changed daily or when visibly soiled. Scrubs and hats worn during dirty or contaminated cases should be changed before subsequent cases, even if not visibly soiled and should be changed at least daily. If scrubs are worn outside the OR within the hospital, they should be covered with a clean lab coat or appropriate cover-up. OR scrubs should not be worn at any time outside the hospital perimeter.

\section{Drapes and gown}

Either sterile, disposable, non-woven or sterile, reusable woven drapes and surgical gowns can be used during surgical operations for the purpose of preventing SSI. However, use of plastic adhesive incise drapes with or without antimicrobial properties does not prevent SSI.

\section{Intraoperative measures}

\section{Skin preparation}

Overall, there is evidence that alcohol-based preparations are more effective in reducing SSI than aqueous preparations, and should be used unless contraindications exist. The rationale for alcohol-based solutions is rapid bactericidal effect, but this benefit is limited by its lack of persistent antimicrobial effect. The addition of iodine-based and chlorhexidine-based solutions prolongs bactericidal activity in alcohol-based preparations. When alcohol preparations are not available, chlorhexidine might be superior to iodine. If diathermy is to be used, ensure that antiseptic skin preparations are dried by evaporation and pooling of alcohol-based preparations is avoided. Currently only povidone-iodine preparations are approved for vaginal surgical site antisepsis. ${ }^{3,4}$

WHO suggests considering the use of wound protector devices in clean-contaminated, contaminated and dirty abdominal surgical procedures for the purpose of reducing the rate of SSI. Wound Protectors are surgical devices which comprise a non-adhesive plastic sheath attached to a single or double rubber ring that firmly secures the sheath to the wound edges. The device is intended to facilitate the retraction of the incision during surgery without the need for additional mechanical retractors and cloths (Figure 1).

\section{Normothermia}

Studies have shown that intraoperative hypothermia is associated with increased risk of SSI, therefore, intraoperative use of warming devices in the operating room for patient body warming is recommended. 
Patients undergoing general anaesthesia with endotracheal intubation should receive an $80 \%$ fraction of inspired oxygen (FiO2) intraoperatively and, if feasible, in the immediate postoperative period for 2-6hours to reduce the risk of SSI. Haemoglobin saturation should be maintained above $95 \%$ (or greater if there is underlying respiratory insufficiency).

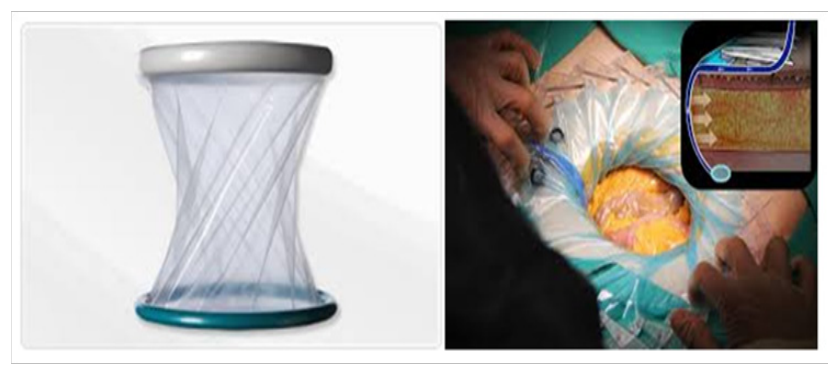

Figure I The device is intended to facilitate the retraction of the incision during surgery without the need for additional mechanical retractors and cloths.

\section{Glycemic control}

Surgery causes a stress response resulting in release of catabolic hormones and the inhibition of insulin making even non-diabetic patients at high risk for hyperglycaemia. Increased blood sugars in the immediate preoperative period is associated with an increased risk of SSI. Optimal blood glucose control should be encouraged for all diabetic patients; however, there is no evidence that improved HbA1C decreases SSI risk. Target perioperative blood glucose should be between 110 to $150 \mathrm{mg} / \mathrm{dL}$ in all patients undergoing surgical procedures to reduce the risk of SSI, regardless of diabetic status. Blood glucose $<110 \mathrm{mg} / \mathrm{dL}$ have been tied to adverse outcomes and increased episodes of hypoglycemia and does not decrease SSI risk.

\section{Wound closure}

Numerous studies have demonstrated decreased risk of SSI with use of triclosan antibiotic suture compared with standard suture and hence, use of triclosan-coated suture is recommended for wound closure in clean and clean-contaminated abdominal cases, when available. Triclosan (5-chloro-2-[2.4- dichlorophenoxy] phenol) is a broad-spectrum bactericidal agent which acts as a bacteriosatic at lower concentrations.

Do not use intracavity lavage to reduce the risk of surgical site infection. There is insufficient evidence to recommend for or against saline irrigation of incisional wounds before closure for preventing SSI. However, irrigation of surgical wound with an aqueous PVP-I solution before closure may be considered, particularly in clean and clean contaminated wounds. Antibiotic incisional wound irrigation before closure should not be used for the purpose of preventing SSI. The panel suggests use of prophylactic negative pressure wound therapy (pNPWT) in adult patients on primarily closed surgical incisions in high-risk wounds (poor tissue perfusion due to surrounding soft tissue/skin damage, decreased blood flow, bleeding/ hematoma, dead space, intraoperative contamination), for preventing SSI, while taking resources into account. When considering different durations of pNPWT (for either $<$ or $>5$ days) and a pressure level (of either $<$ or $>100 \mathrm{mmHg}$ ), the observed benefit with the use of pNPWT remained unchanged.

Do not apply antimicrobial agents (ie, ointments, solutions, or powders) to the surgical incision for the prevention of SSI. During the operation, glove decontamination with alcohol or other products for the purpose of reuse should never be performed. In the case of doublegloving, a routine change of the outer gloves during long surgeries is often recommended by health care practitioners. However, no evidence was found to support these practices. Changing outer gloves and using new instruments for closure after contaminated surgical procedures seems logical, particularly after colorectal surgery or in patients operated for diffuse peritonitis represent common-sense practices that have become convention.

Tissue adhesives are the most expensive closure material but generate cost savings when compared with sutures owing to shorter time for wound closure and no need for a postoperative outpatient visit. Wound closure with adhesive tape is cheaper compared to adhesives or sutures; adhesive tape is faster to apply and less costly. Sutures require the greatest time for wound closure and also require a postoperative visit for removal. However, sutures are less expensive than clips.

\section{Postoperative measures}

\section{Surgical antibiotic prophylaxis (SAP) prolongation}

The current recommendation is that all prophylactic antibiotics be terminated within 24hours of surgery completion, because there is no documented benefit in reduction of surgical site infection after skin closure. Antibiotics should be continued only when clear medical indications are present. There is a risk of promoting antimicrobial resistance if antibiotics are prolonged postoperatively, both at the individual level and at the health care facility level. In addition, this practice might negatively affect the patient microbiome and lead to short- and long-term gastrointestinal complications, including intestinal C. Difficile infection. Perioperative antibiotic prophylaxis should not be continued in the presence of a wound drain to prevent SSI. The wound drain should be removed when clinically indicated and no optimal timing for its removal has been recommended.

\section{Wound dressings}

The main purpose of surgical dressings is to allow appropriate assessment of the wound postoperatively, to absorb exudates, to ease pain and to provide protection for newly formed tissue. They maintain an optimal moist wound environment without causing maceration of the surrounding skin as the dressing material is permeable to moisture and gas. Use of gauze as a primary dressing should be avoided because of its association with pain and disruption of healing tissues at the time of dressing change. Surgical incisions should be covered with an appropriate interactive dressing at the end of the operation.

Surgical dressings should be kept undisturbed for a minimum of 48hours after surgery unless leakage occurs. For contaminated wounds closed with interrupted staples, daily wound probing resulted in lower SSI rate and decreased length of stay without increased patient discomfort, and should be considered for this wound class.

Use sterile saline for wound cleansing up to 48hours after surgery. The patients may shower safely 48 hours after surgery. Do not use topical antimicrobial agents for surgical wounds that are healing by primary intention to reduce the risk of SSI. Use tap water for wound cleansing after 48hours if the surgical wound has separated or has been surgically opened to drain pus. Do not use Eusol and gauze, or moist cotton gauze or mercuric antiseptic solutions to manage surgical 
wounds that are healing by secondary intention and an appropriate interactive dressing should be used.

\section{Dressing materials may be classified as:}

A. Passive - such as 'gauze-like materials' that simply cover the wound, neither promoting nor intentionally hindering the wound healing process. They have been associated with negative effects on the patient's quality of life during the 30day postoperative period.

B. Interactive - modern dressing materials that are designed to promote the wound healing process through the creation and maintenance of a local, warm, moist environment underneath the chosen dressing, when left in place for a period indicated through a continuous assessment process. Examples are alginates, semi-permeable film membranes, foams, hydrocolloids and fibrous hydrocolloids, etc.

C. Active - These are designed to manipulate or alter the wound healing environment to either re-stimulate or to further promote the wound healing process. Examples include topical negative pressure therapy, larva therapy (sterile maggots), dressings con- taining antimicrobial agents or biomaterials such as collagen or hyaluronic acid or cultured keratinocytes or bio-engineered skin.

\section{Acknowledgements}

None.

\section{Conflict of interest}

Author declares that there is no conflict of interest.

\section{References}

1. WHO. Global Guidelines for the Prevention of Surgical Site Infection Geneva: World Health Organization; 2016.

2. Berríos-Torres SI, Umscheid CA, Bratzler DW, et al. Centers for Disease Control and Prevention Guideline for the Prevention of Surgical Site Infection, 2017. JAMA Surg. 2017;152(8):784-791.

3. NICE Guideline. Surgical site infections: prevention and treatment. 2017:(CG74)

4. Ban KA, Minei JP, Laronga C, et al. American College of Surgeons and Surgical Infection Society: Surgical Site Infection Guidelines, 2016 Update. J Am Coll Surg. 2017;224(1):59-74. 